Research Paper

\title{
P53 Is Involved in a Three-Dimensional Architecture- Mediated Decrease in Chemosensitivity in Colon Cancer
}

\author{
Jianming $\mathrm{He}^{1 \bowtie *}$, Xi Liang1,2* ${ }^{1,}$ Fen $\mathrm{Luo}^{2}$, Xuedan $\mathrm{Chen}^{3}$, Xueqing $\mathrm{Xu}^{2}$, Fengchao Wang4, Zhenping Zhang ${ }^{5}$ \\ 1. Department Of Oncology And Southwest Cancer Center, Southwest Hospital, Third Military Medical University, Chongqing, 400038 China; \\ 2. Molecular Biology Center, State Key Laboratory of Trauma, Burn, and Combined Injury, Daping Hospital, Third Military Medical University, Chongqing, \\ 400042 China; \\ 3. Department Of Medical Genetics, Third Military Medical University, Chongqing, 400038 China; \\ 4. Institute of Combined Injury, State Key Laboratory of Trauma, Burns and Combined Injury, Third Military Medical University, Chongqing, 400038 China; \\ 5. Department Of Oncology, First Hospital of Shijiazhuang City, Shijiazhuang, Hebei Province, 050011 China. \\ *These authors contributed equally to this manuscript. \\ $\triangle$ Corresponding author: Tel: +86 23 68773057; Email: hejm@tmmu.edu.cn (J. He).
}

(C) Ivyspring International Publisher. Reproduction is permitted for personal, noncommercial use, provided that the article is in whole, unmodified, and properly cited. See http://ivyspring.com/terms for terms and conditions.

Received: 2015.11.22; Accepted: 2016.03.16; Published: 2016.04.29

\begin{abstract}
Three-dimensional (3D) culture models represent a better approximation of solid tumor tissue architecture, especially cell adhesion, in vivo than two-dimensional (2D) cultures do. Here, we explored the role of architecture in chemosensitivity to platinum in colon cancer. Under the 3D culture condition, colon cancer cells formed multicellular spheroids, consisting of layers of cells. 3D cultures displayed significantly decreased sensitivity to platinum compared with 2D cultures. Platinum increased $\mathrm{p} 53$ in a dose-dependent and time-dependent manner. There was no detectable difference in basal $\mathrm{p} 53$ levels between $3 \mathrm{D}$ cultures and $2 \mathrm{D}$ cultures but cisplatin induced less p53 in both HCT116 3D cultures and LoVo 3D cultures. It was not due to cisplatin concentration because cisplatin induced similar $\mathrm{Y}-\mathrm{H} 2 \mathrm{AX}$ in 3D vs 2D. Knockdown of p53 significantly decreased sensitivity to platinum in 3D cultures. Knockdown of $\mathrm{p} 53$ decreased cleaved caspase 3 and apoptosis induced by cisplatin. These findings indicate that 3D architecture confers decreased chemosensitivity to platinum and $p 53$ is involved in the mechanism. Knockdown of $p 53$ decreased cisplatin's induction of c-Jun $\mathrm{N}$-terminal kinase $1 / 2(\mathrm{JNK} 1 / 2)$ activation, whereas inhibition of JNK1/2 activation increased chemosensitivity. Inhibition of $\mathrm{p} 38$ activation decreased cisplatin's induction of $\mathrm{p} 53$, but no difference in $\mathrm{p} 38$ activation by cisplatin was observed between $2 \mathrm{D}$ cultures and 3D cultures. Taken together, our results suggest that $\mathrm{p} 53$ is involved in a 3D architecture-mediated decrease in chemosensitivity to platinum in colon cancer. Mitogen-activated protein kinases (JNK1/2 and p38) do not play a dominant role in the mechanism.
\end{abstract}

Key words: p53; platinum; three-dimensional culture; colorectal cancer cells.

\section{Introduction}

Colorectal cancer is the third most commonly diagnosed cancer in males and the second most in females worldwide, with an estimated 1.4 million cases occurring in 2012[1]. The incidence of colorectal cancer in developing countries is increasing, which is partly attributable to lipid metabolism [1-2]. Colorectal cancer is the fourth most lethal disease among all malignancies in males and the third most in females, with an estimated 693,900 deaths occurring in 2012[1]. Platinum (including cisplatin (CDDP), oxaliplatin (L-OHP), nedaplatin and so on) remains one of the most effective and most commonly used drugs to treat this disease [3]. Unfortunately, resistance to chemotherapy remains a critical barrier 
to the development of an effective cure [1, 3-4]. More than $35 \%$ of colorectal cancer patients die within 5 years after diagnosis, even in developed countries such as the United States [4].

Most in vitro experimental studies of anticancer drugs testing and resistance mechanisms are performed with monolayer cell cultures (two-dimensional (2D) cell cultures) [5-6]. 2D cell cultures are not an optimal experimental system for studies of anticancer drugs because these cultures do not well recapitulate solid tumor tissue architecture in vivo, and the architecture is important for tumor cells' biological behaviors, including chemosensitivity [7-10]. Three-dimensional (3D) cell cultures represent a better approximation of solid tumor tissue architecture than 2D cultures do [7-12].

A few researchers have reported that certain types of tumor cells in 3D cultures are more sensitive to specific anticancer drugs than in 2D cultures[13], but most researchers believe that most tumor cells in 3D cultures are more resistant to anticancer drugs[7, $9-11,14]$. The differences in sensitivities to most drugs are not attributed to drug concentrations because drug penetration and delivery in 3D cultures has been shown to be negligible for most anticancer drugs, including platinum, 5-fluorouracil, carmustine, lomustine, chlorambucil, bleomycin, and mitomycin, among others [9-10].

In the present study, we explored the influence of architecture on the response of human colorectal cancer cells to platinum using both $2 \mathrm{D}$ cultures and $3 \mathrm{D}$ cultures. Our data demonstrate that 3D architecture conferred decreased chemosensitivity to platinum, by or partly by weaker p53 induction.

\section{Materials and Methods}

\section{Cell lines and cell culture}

The human colorectal cancer cell lines HCT116, LoVo and the human embryonic kidney cell line 293T were obtained from the Cell Bank of the Chinese Academy of Science.

2D cultures were routinely grown and passaged as previously described [7]. In brief, cells were grown in McCoy's 5A (Gibco, Grand Island, NY, USA, Cat: 16600108) (HCT116), F12K (Gibco, Cat: 12500-062) (LoVo) or DMEM (Gibco, Cat: 12100-046) (293T) supplemented with $100 \mathrm{ml} / \mathrm{L}$ fetal bovine serum (Gibco, Cat: 10438018), 100,000 IU/L penicillin, and $100 \mu \mathrm{g} / \mathrm{ml}$ streptomycin (Gibco, Cat: 15140122) under a humidified atmosphere of $5 \% \mathrm{CO}_{2}$ at $37^{\circ} \mathrm{C}$.

$3 \mathrm{D}$ cultures were prepared by using the liquid overlay technique as previously described [7-8]. In brief, exponentially growing cancer cells were seeded into plates that had been previously coated with $2 \%$ agarose. The plates were then gently horizontally swirled for 10 minutes every 6 hours for the first 24 hours. The cells were incubated under a humidified atmosphere of $5 \% \mathrm{CO}_{2}$ at $37^{\circ} \mathrm{C}$. The medium was appropriately refreshed every day.

\section{Hematoxylin and eosin (H\&E) staining}

HCT116 3D cultures were fixed in $4 \%$ paraformaldehyde, and OCT-embedded samples were sectioned at a thickness of $10 \mu \mathrm{m}$. Sample slides were routinely stained with $\mathrm{H} \& \mathrm{E}$ [12].

LoVo 3D cultures were fixed in $4 \%$ paraformaldehyde, and paraffin-embedded samples were sectioned at a thickness of $6 \mu \mathrm{m}$. Sample slides were routinely stained with H\&E [15].

\section{Slides preparation for transmission electron microscopy}

Sample slides were routinely prepared as previously described $[7,11]$. In brief, 3D cultures were fixed in $2.5 \%$ glutaraldehyde and then in $1 \%$ osmium tetroxide. Samples were dehydrated using graded alcohol, and ultrathin sections were generated. The sections were stained with uranium acetate and lead citrate and observed using a transmission electron microscope (TECNAI10, Philip, Holland).

\section{Lentiviral delivery of shRNA}

p53 was knocked down by lentiviral vector-mediated small hairpin (sh) RNA interference using The RNAi Consortium system (Open Biosystems, Inc., Huntsville, AL, USA) according to the manufacturer's instructions[6, 11]. In brief, a p53-targeting shRNA-pLKO.1 vector or a control shRNA-pLKO.1 vector, with the packaging plasmid pCMV-Dr8.91 and the enveloping plasmid pCMV-VSV-G, was co-transfected into 293T cells using Lipofectamine ${ }^{\circledR} 2000$ (Invitrogen, Carlsbad, CA, USA, Cat: 11668-030) according to the manufacturer's instructions. Virus-containing medium was collected at 48 and 72 hours post-transfection and was filtered. Cells were infected with lentivirus encoding the shRNA targeting p53 or with control lentivirus and were then selected using puromycin (Sigma-Aldrich, St. Louis, MO, USA, Cat: P8833). The sense sequence of the shRNA targeting p53 is GACTCCAGTGGTAATCTACT[6]. Control shRNA (shcontrol) is targeted against green fluorescent protein, and the sense sequence of this shRNA is TACAACAGCCACAACGTCTAT[11]. Knockdown efficiency was confirmed by western blot.

\section{Water-soluble tetrazolium salt (WST) assay}

Cells were treated with $50 \mu \mathrm{g} / \mathrm{ml}$ CDDP or 20 $\mu \mathrm{g} / \mathrm{ml} \mathrm{L}-\mathrm{OHP}$ for 24 hours. Control cultures received $10 \mu 1$ PBS (Fig. 1C and 3F) or SP600125 (Fig. 4C) only. 
Cell viability was assayed using WST (2-(2-methoxy-4-nitrophenyl)-3-(4-nitrophenyl)-5-(2,4 -disulfophenyl)-2H-tetrazolium, monosodium salt). The WST assay was performed using Cell Counting Kit 8 (Dojindo Laboratories, Kumamoto, Japan, Cat: CK04) according to the manufacturer's instructions, as previously described [7, 12]. In brief, 2D cultures or 3D cultures detached using accutase were incubated with WST/media for 2-4 hours, after which the absorbance at $450 \mathrm{~nm}$ was measured using a microplate reader with a reference wavelength of 650 $\mathrm{nm}$. Cell viability was normalized to the control.

\section{Clonogenic assay}

Clonogenic assay in vitro was routinely grown and passaged as previously described [11-12]. In brief, the same numbers of cells were treated with $50 \mu \mathrm{g} / \mathrm{ml}$ CDDP for 24 hours. Then, the 3D cultures were detached using accutase, and the same ratio of single-cell suspensions was seeded into 24 -well plates in duplicate. The cells were cultured at $37^{\circ} \mathrm{C}$ for 7 days and were subsequently stained with crystal violet.

\section{Immunohistochemical staining}

HCT116 3D cultures were fixed in $4 \%$ paraformaldehyde, and OCT-embedded samples were sectioned at a thickness of $10 \mu \mathrm{m}$. Immunohistochemistry was performed according to the protocol of the SPlink Detection Kits (ZSGB-BIO, Beijing, China, Cat: SP-9000), as previously described $[12,15]$.

\section{Apoptosis assay}

shcontrol HCT116 3D cultures and shp53 HCT116 3D cultures were treated with $20 \mu \mathrm{g} / \mathrm{ml}$ CDDP for 24 hours and then fixed in $4 \%$ paraformaldehyde. OCT-embedded samples were sectioned at a thickness of $10 \mu \mathrm{m}$. The terminal deoxynucleotidyl transferase-mediated nick end-labeling (TUNEL) stain was used to detect DNA fragmentation resulting from apoptosis via an In Situ Cell Death Detection Kit, Fluorescein (Roche, Germany, Cat: 11684795910), according to the manufacturer's instructions, as described previously [7].

\section{Preparation of cell lysates}

Cells were lysed in RIPA buffer $(50 \mathrm{mM}$ Tris base, $150 \mathrm{mM} \mathrm{NaCl}, 1 \%$ Nonidet P-40, 0.25\% Na-deoxycholate, $1 \mathrm{mM}$ EDTA) with protease inhibitors and phosphatase inhibitors (1 mM PMSF, 5 $\mu \mathrm{g} / \mathrm{ml}$ leupeptin, $2 \mu \mathrm{g} / \mathrm{ml}$ pepstatin, $4 \mu \mathrm{g} / \mathrm{ml}$ aprotinin, $10 \mathrm{mM} \mathrm{NaF}, 1 \mathrm{mM} \mathrm{Na} 3 \mathrm{VO} 4$, and $10 \mathrm{mM}$ $\beta$-glycerophosphate disodium salt pentahydrate) for 30 minutes on ice. Following centrifugation $(26,000 \mathrm{~g}$,
16 minutes, $4^{\circ} \mathrm{C}$ ), the supernatant was collected as total cell protein [11-12].

\section{Western blot analysis}

The protein was resolved by SDS/PAGE and blotted on nitrocellulose membranes (Bio-Rad, Richmond, CA, USA, Cat: 162-0115) as previously described $[2,7]$. The nitrocellulose membranes were incubated with specific primary antibodies overnight. After incubation with secondary antibodies, immunoreactive proteins were visualized using the Enhanced Chemiluminescent Substrate (Thermo Scientific, Pittsburgh, PA, USA, Cat: 34095).

Primary antibodies against cleaved caspase 3 (Cat: 9664, 1:500), p53 (Cat: 2524, 1:1000), phospho-p53 (Cat: 9286, 1:500), $\beta$-catenin (Cat: 9582, 1:1000), $\gamma$-H2AX (Cat: 9718, 1:1000), a-tubulin (Cat: 3873, 1:1000), $\beta$-actin (Cat: 3700, 1:1000), Histone H3 (Cat: 3638, 1:1000) and glyceraldehyde 3-phosphate dehydrogenase (GAPDH) (Cat: 8884, 1:1000); the phospho-mitogen-activated protein kinase (MAPK) Family Antibody Sampler Kit (Cat: 9910, 1:1000); and HRP-linked secondary antibodies were from Cell Signaling Technology (Beverly, MA, USA).

\section{MAPK activation inhibitors}

The c-Jun N-terminal kinase (JNK) inhibitor SP600125 [16] (Cat: S1460) and the p38 inhibitor SB202190 [17] (Cat: S1077) were from Selleck Chemicals (Houston, TX, USA).The MAPK/ extracellular signal-regulated kinase (ERK) kinase 1/2 inhibitor U0126 [18] (Cat: 9910) was from Cell Signaling Technology.

\section{Statistical analyses}

The data shown represent the mean \pm standard error. Statistical differences between groups were analyzed by Student's t-test or one-way ANOVA. $p<$ 0.05 was considered statistically significant.

\section{Results}

\section{D architecture confers decreased sensitivity to platinum in colorectal cancer}

The phenotypes of the colon cancer cell lines HCT116 and LoVo when cultured as 2D and 3D cultures were analyzed. Colon cancer cells cultured on plastic (2D) grew as flat monolayers (Fig. 1A and B). When maintained under the 3D culture condition, colon cancer cells formed multicellular spheroids (Fig. $1 \mathrm{~A}$ and $\mathrm{B})$. H\&E staining showed that the multicellular spheroids consisted of layers of cells. Necrotic core was not found (Fig. 1A and B). By employing a transmission electron microscope, cell-cell junctions were found in most cells in HCT116 3D culture (Fig. S1). 

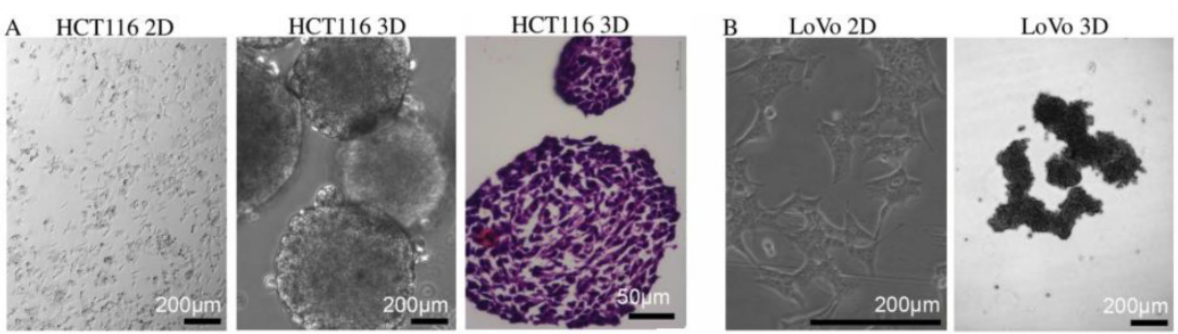
LoVo 3D
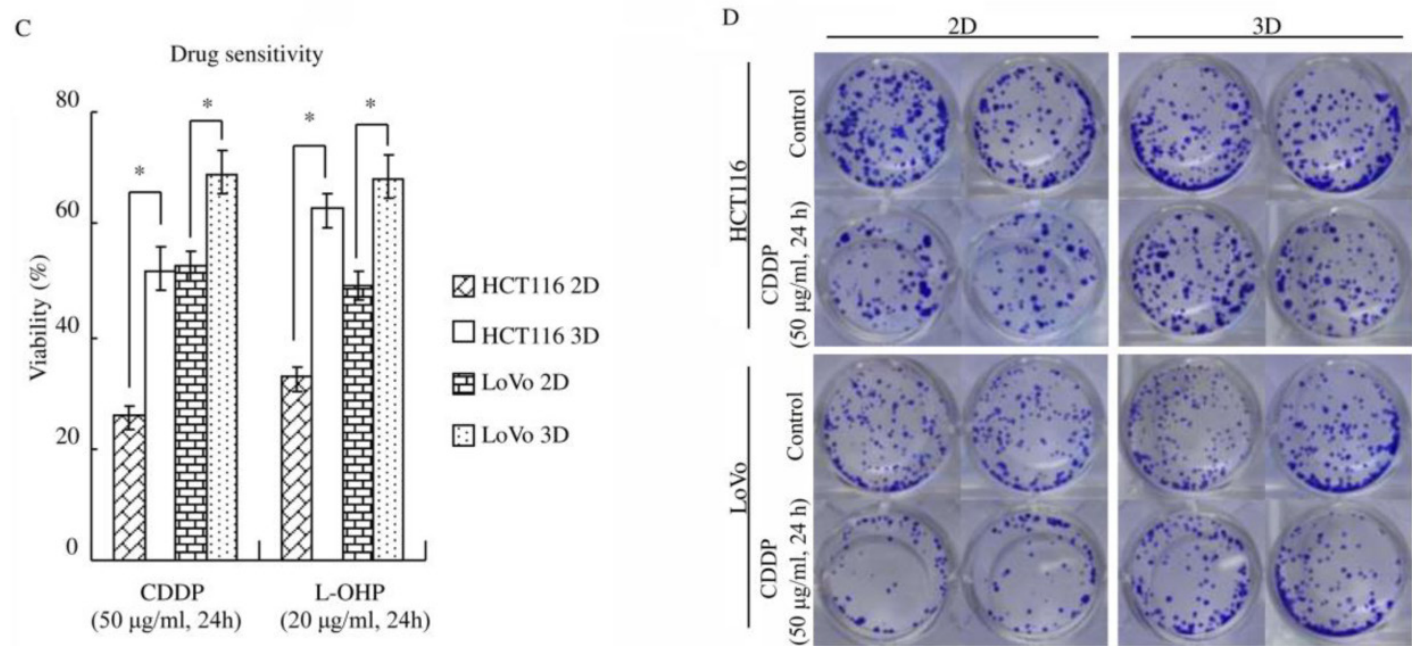

Figure 1. 3D architecture confers decreased sensitivity to platinum in colorectal cancer. (A-B) HCT116 and LoVo cultured on plastic grew as flat monolayers (left) and formed multicellular spheroids (middle) using the liquid overlay technique. Multicellular spheroids sections were stained with H\&E (right). (C) WST assay indicated that $3 D$ cultures were less sensitive to platinum compared with 2D cultures. $*: p<0.01$ (D) After 3D or 2D cultures treated with or without CDDP, cells were detached and cultured as monolayer cells for 7 days and were subsequently stained with crystal violet. The clonogenicity of the 3D cultures treated with CDDP was much higher than that of the respective 2D cultures.

To explore the role of $3 \mathrm{D}$ architecture in chemosensitivity, cells were treated with platinum because it was well elucidated that platinum penetration and delivery in 3D cultures is negligible [9-10]. Cell viability was assayed using WST. 3D cultures displayed a significant decrease in chemosensitivity compared with $2 \mathrm{D}$ cultures $(p<0.01)$ (Fig. 1C). The cell viability of HCT116 3D cultures treated with CDDP was $51.8 \pm 3.9 \%$, whereas that of $2 \mathrm{D}$ cultures was $25.4 \pm 1.8 \%(p<0.01)$. The cell viability of HCT116 3D cultures treated with L-OHP was $62.7 \pm 3.0 \%$, whereas that of $2 \mathrm{D}$ cultures was $32.3 \pm 2.7 \%$ $(p<0.01)$. For LoVo cells, the viability of 3D cultures treated with CDDP was $69.1 \pm 3.7 \%$, whereas that of $2 \mathrm{D}$ cultures was $52.4 \pm 2.7 \%(p<0.01)$. The cell viability of LoVo 3D cultures treated with L-OHP was $68.3 \pm 4.1 \%$, whereas that of $2 \mathrm{D}$ cultures was $48.9 \pm 2.8 \% \quad(p<0.01)$. The results of the clonogenic assay were consistent with those of the WST assay. The clonogenicity of the HCT116 3D cultures and LoVo 3D cultures was much higher than that of the respective 2D cultures (Fig. 1D).

\section{Weaker p53 induction contributes to the 3D architecture-mediated decrease in chemosensitivity}

Platinum kills cells by damaging DNA and p53 plays a key role in the DNA damage response [19-21]. Therefore, the role of p53 in the 3D architecture-mediated decrease in sensitivity to platinum was explored.

Platinum increased the p53 protein level in both HCT116 and LoVo cells (both of them contain stabilized wild-type p53 protein [22-23]), regardless of whether the cells were in 2D or 3D cultures (Fig. 2). p53 protein was increased by platinum in a dose-dependent and time-dependent manner (Fig. 2). There was no detectable difference in basal p53 levels between 3D cultures and 2D cultures but CDDP induced less p53 in both HCT116 3D cultures and LoVo 3D cultures. (Fig. 2C and D).

Although it was well elucidated that platinum penetration and delivery in 3D cultures is negligible [9-10], $\mathrm{Y}-\mathrm{H} 2 \mathrm{~A} . \mathrm{X}$, a maker of DNA damage [24], was assayed by western blot to confirm this aspect. CDDP induced similar $\mathrm{\gamma}-\mathrm{H} 2 \mathrm{AX}$ protein in HCT116 3D cultures as in 2D cultures (Fig. 2E). This finding 
suggests that $3 \mathrm{D}$ architecture does not decrease the concentration of CDDP to cells in 3D cultures.

Following DNA damage, cells activate the DNA damage response, which either leads the cell to repair the lesion, promoting resistance to platinum, or causes cell death due to activation of the apoptotic response [19-20, 25]. p53 has been shown to play a key role in the DNA damage response in that p53 can initiate DNA repair; cell cycle arrest; senescence; and, notably, apoptosis[19-20, 25]. Hence, the role of p53 and apoptosis in 3D architecture-mediated decreased sensitivity to platinum was explored.

p53 was knocked down using The RNAi Consortium system. The efficiency and specificity of the sequence of the shRNA targeting p53 were previously verified [6]. Western blot showed that lentiviral delivery of the shRNA targeting p53 efficiently knocked down p53 in HCT116 3D cultures and that shcontrol did not detectably change the p53 protein level (Fig. 3A and B). Immunohistochemistry showed that p53 was expressed uniformly in cells in HCT116 3D cultures and that knockdown of p53 uniformly decreased its expression in cells (Fig. 3C). Western blot also showed that the system efficiently decreased CDDP's induction of p53 and p-p53. Knockdown of p53 significantly decreased cleaved caspase 3, which plays a central role in the execution phase of cell apoptosis, in 3D cultures (Fig. 3D and E) and 2D cultures (Fig. 3F)[26]. To confirm that knockdown of p53 decreased apoptosis induced by platinum treatment, TUNEL staining was used to detect apoptosis in HCT116 3D cultures. Treatment with $20 \mu \mathrm{g} / \mathrm{ml} \mathrm{CDDP}$ for 24 hours clearly induced less apoptosis in HCT116 3D cultures with p53 knockdown than in shcontrol HCT116 3D cultures (Fig. 3G).

Inducing apoptosis is an important way of platinum killing cells by DNA damage [19-21]. Because knockdown of p53 decreased apoptosis induced by platinum, knockdown of p53 might decrease sensitivity to platinum. To validate this hypothesis, chemosensitivity was assayed using WST. Knockdown of p53 significantly decreased chemosensitivity $(p<0.01)$ (Fig. $3 \mathrm{H})$. The cell viability of shcontrol HCT116 3D cultures treated with CDDP was $48.6 \pm 3.7 \%$, whereas that of treated HCT116 cells with p53 knockdown was $64.4 \pm 3.1 \%(p<0.01)$. The cell viability of shcontrol HCT116 3D cultures treated with L-OHP was $62.0 \pm 2.9 \%$, whereas that of treated HCT116 cells with p53 knockdown was $73.0 \pm 2.1 \%$ $(p<0.01)$.
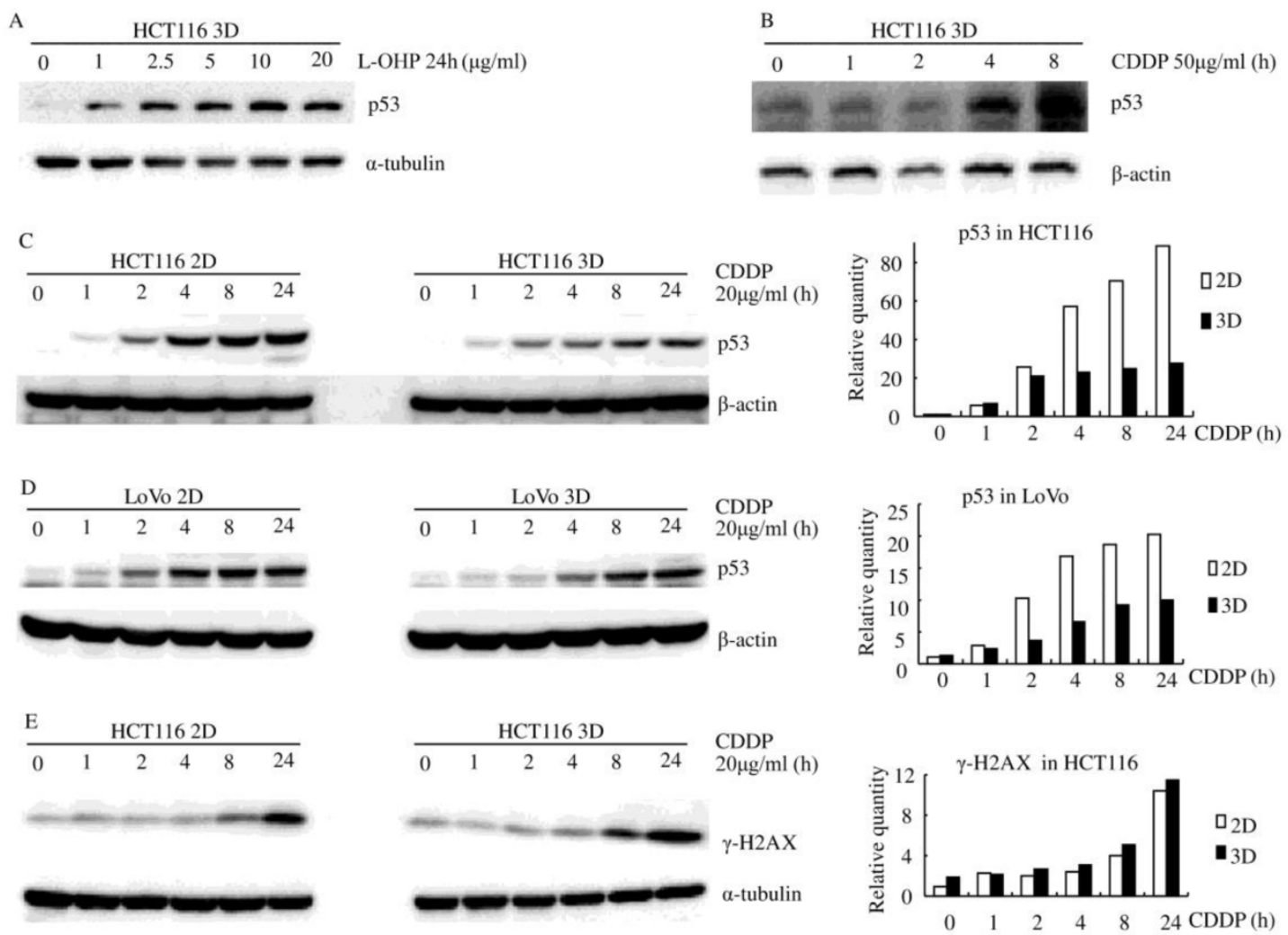

Figure 2. Platinum induces less $\mathrm{p} 53$ in 3D architecture (western blot). (A) L-OHP induced p53 in a dose-dependent manner. (B) CDDP induced $\mathrm{p} 53$ in a time-dependent manner. (C-D) CDDP induced less p53 in 3D cultures than in the respective 2D cultures. Relative quantities of $p 53$ were normalized to $\beta$-actin and diagramed, respectively (right). (E) CDDP induced similar $\mathrm{y}-\mathrm{H} 2 \mathrm{~A} . \mathrm{X}$ protein in $\mathrm{HCT} 1163 \mathrm{D}$ cultures as in $2 \mathrm{D}$ cultures. Relative quantities of $\mathrm{p} 53$ were normalized to $\alpha$-tubulin and diagrammed (right). 
A

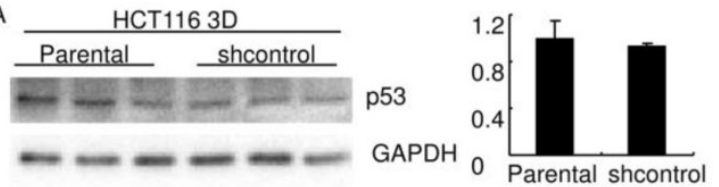

B

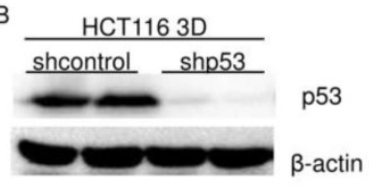

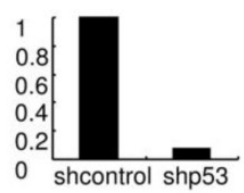

C

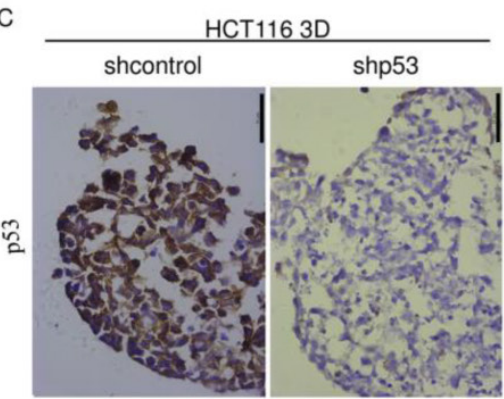

D HCT116 3D+CDDP $(20 \mu \mathrm{g} / \mathrm{ml}, 24 \mathrm{~h})$
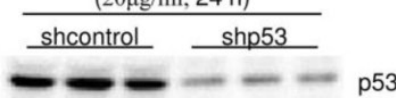

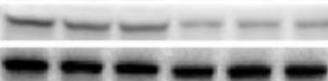

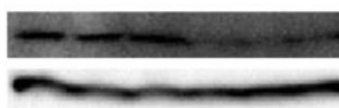

Histone $\mathrm{H} 3$

E HCT116 3D+CDDP
$(20 \mu \mathrm{g} / \mathrm{ml}, 48 \mathrm{~h})$

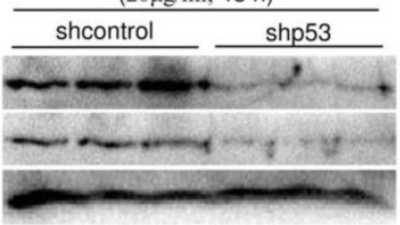

53

cleaved caspase 3

Histone $\mathrm{H} 3$

F

HCT116 2D+CDDP

$(20 \mu \mathrm{g} / \mathrm{ml}, 24 \mathrm{~h})$

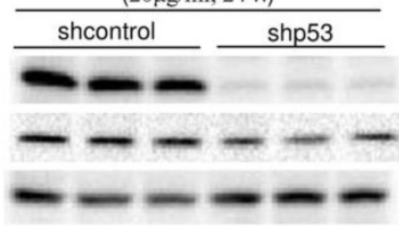

p53

cleaved caspase 3

$\beta$-actin

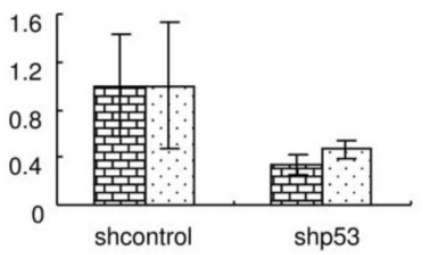

shcontrol

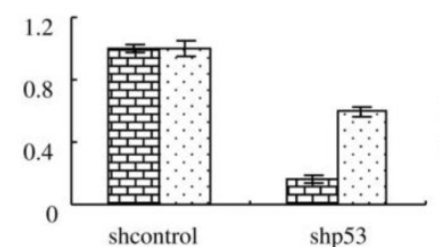

回 553

$\square$ cleaved

caspase 3

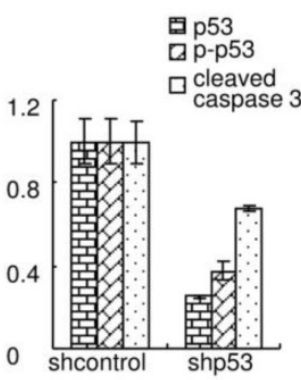

G

TUNEL

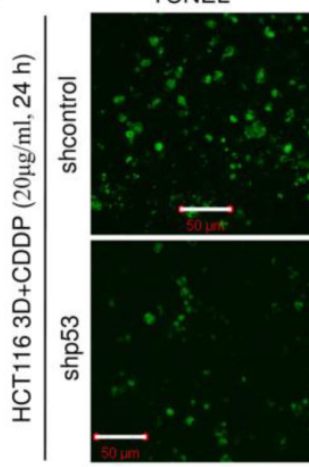

DAPI

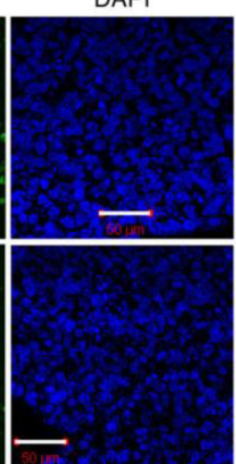

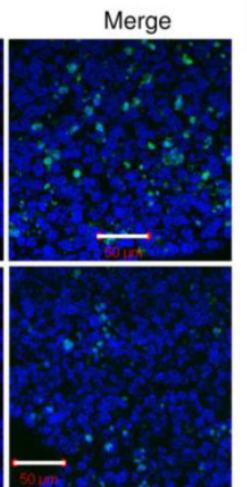

Drug sensitivity of HCT116 3D

Figure 3. Knockdown of p53 decreases sensitivity to platinum in HCT116. (A) There was no detectable difference in the p53 protein level between Parental and shcontrol cells. Relative quantities of p53 were normalized to GAPDH and diagrammed (right). (western blot) (B) The protein p53 was knocked down by lentiviral delivery of shRNA (shp53), and the efficiency was assayed by western blot. Relative quantities of p53 were normalized to $\beta$-actin and diagrammed (right). (C) Immunohistochemical staining of p53 in HCT116 3D cultures. (D) After treatment of CDDP for 24 hours, p53, p-p53 and cleaved caspase 3 in 3D cultures were assayed by western blot. Relative quantities were normalized to their loading control and diagrammed, respectively (right). (E) After treatment of CDDP for 48 hours, p53 and cleaved caspase 3 in $3 D$ cultures were assayed by western blot. Relative quantities were normalized to Histone $\mathrm{H} 3$ and diagrammed (right). (F) After treatment of CDDP for 24 hours, p53 and cleaved caspase 3 in $2 \mathrm{D}$ cultures were assayed by western blot. Relative quantities were normalized to $\beta$-actin and diagrammed (right). (G) After treatment of CDDP for 24 hours, apoptosis was detected with TUNEL stain. $(\mathrm{H})$ Cell viabilities of HCT116 3D cultures were assayed by WST. *:p<0.01. 
A
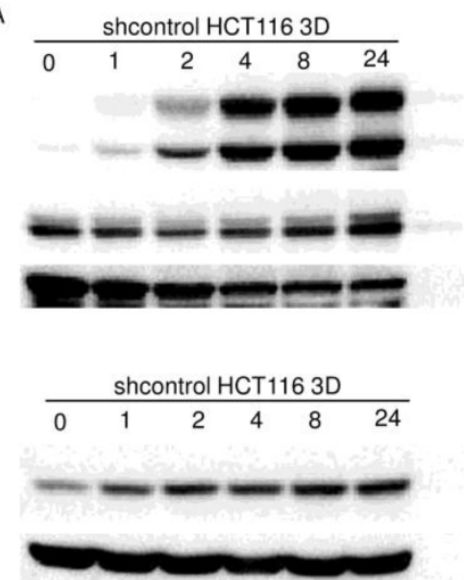

B

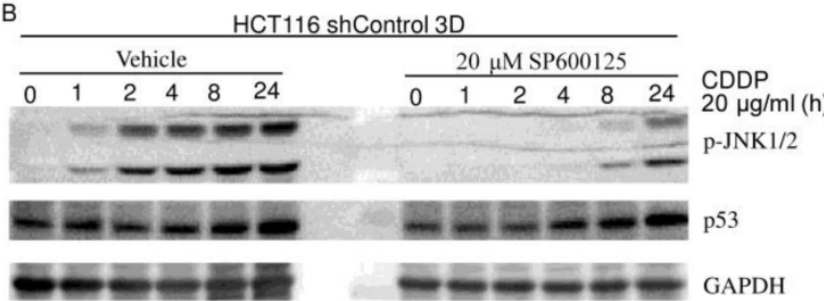

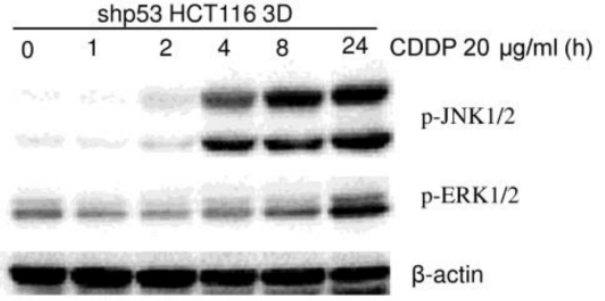

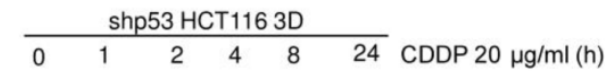

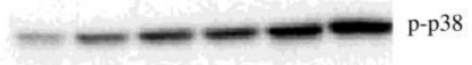

$\beta$-actin

C Drug sensitivity of HCT116 3D

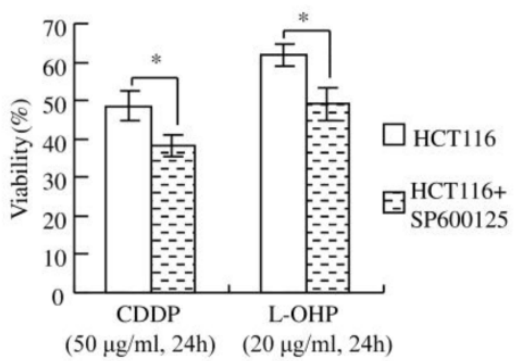

Figure 4. JNK1/2 do not play a dominant role in the 3D architecture-mediated p53-dependent decrease in chemosensitivity. (A) MAPKs (JNK1/2, $\mathrm{p} 38$ and ERK 1/2) were activated by CDDP in a time-dependent manner. Knockdown of $\mathrm{p} 53$ decreased CDDP's activation of JNK1/2, but not $\mathrm{P} 38$ or ERK1/2 (western blot). (B) Cells were treated with vehicle or SP600125 1 hour prior to CDDP treatment. CDDP's activation of JNK1/2 was remarkably inhibited by SP600125. Inhibition of JNK1/2 activation did not detectably change CDDP's induction of p53 (western blot). (C) Cells were treated with vehicle or SP600125 1 hour prior to CDDP treatment. Cell viability was assayed using WST. Pretreatment with $20 \mu \mathrm{M}$ SP600125 significantly increased chemosensitivity. *:p<0.05.

\section{MAPKs (JNK1/2, p38 and ERK1/2) do not play a dominant role in the $3 \mathrm{D}$ architecture- mediated p53-dependent decrease in chemosensitivity}

MAPKs play a remarkable role in apoptosis [5, 16, 18, 27-28]. JNK1/2, p38 and ERK1/2, three groups of MAPKs, have been reported to be related to platinum resistance and p53 [20-21, 29-30]. Here, the roles of JNK1/2, p38 and ERK1/2 in the 3D architecture-mediated p53-dependent decrease in chemosensitivity were explored.

HCT116 3D cultures were treated with $20 \mu \mathrm{g} / \mathrm{ml}$ CDDP for different amounts of time. Western blot showed that JNK1/2 was activated in a time-dependent manner (Fig. 4A and B). Knockdown of p53 decreased CDDP's activation of JNK1/2 in HCT116 3D cultures (Fig. 4A). CDDP's activation of JNK1/2 was remarkably inhibited by $20 \mu \mathrm{M}$ SP600125 treatment 1 hour prior to CDDP treatment. Inhibition of JNK1/2 activation did not detectably change CDDP's induction of p53 in HCT116 3D cultures (Fig. 4B). These results indicate that p53 activates JNK1/2 in response to CDDP treatment, and this led to the hypothesis that JNK1/2 might play a role in the 3D architecture-mediated p53-dependent decrease in chemosensitivity. Then, chemosensitivities of HCT116 shcontrol 3D cultures were assayed. The result was opposite to our hypothesis: pretreatment with $20 \mu \mathrm{M}$ SP600125 1 hour prior to platinum treatment significantly increased chemosensitivity $(P<0.05)$ (Fig. 4C). The cell viability of shcontrol HCT116 3D cultures pretreated with SP600125 prior to CDDP was $38.4 \pm 2.8 \%$, and that of shcontrol HCT116 3D cultures pretreated with SP600125 prior to L-OHP was $49.1 \pm 4.4 \%$. These findings indicate that JNK $1 / 2$ do not play a dominant role in the $3 \mathrm{D}$ architecture-mediated p53-dependent decrease in chemosensitivity.

To explore the role of p38 in the 3D architecture-mediated p53-dependent decrease in sensitivity to platinum, both HCT116 3D cultures and LoVo 3D cultures were treated with CDDP for different amounts of time or were treated with a gradient of L-OHP for 24 hours. p-p38 protein was then analyzed by western blot. p38 was activated by platinum treatment in a dose-dependent and time-dependent manner (Fig. 4A, 5, 6A and 6B). Knockdown of p53 did not detectably change the p-p38 level induced by CDDP treatment (Fig. 4A). CDDP's activation of p38 was remarkably inhibited by $20 \mu \mathrm{M}$ SB202190 treatment 1 hour prior to CDDP (Fig. 6A). Though inhibition of p38 activation decreased CDDP's induction of p53 (Fig. 6A), there was no detectable difference in $\mathrm{CDDP}^{\prime} \mathrm{s}$ induction of 
p-p38 between HCT116 2D cultures and 3D cultures (Fig. 6B). These findings suggest that p38 does not play a role in the $3 \mathrm{D}$ architecture-mediated p53-dependent decrease in chemosensitivity.

The role of ERK1/2 in the 3D architecture-mediated p53-dependent decrease in chemosensitivity was explored in a similar way as for p38. ERK1/2 was activated in a dose-dependent and time-dependent manner by platinum treatment (Fig. 4A, 5 and 6C). Knockdown of p53 did not detectably

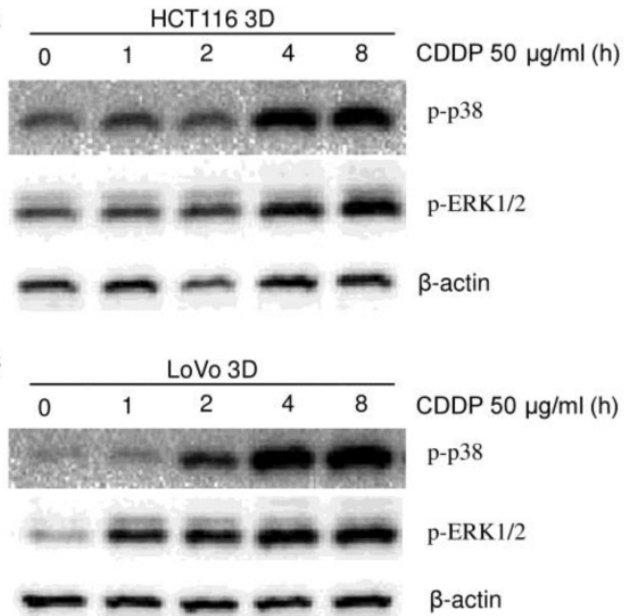

change the $p$-ERK1/2 level induced by CDDP treatment (Fig. 4A). CDDP's induction of p-ERK1/2 was remarkably inhibited by $20 \mu \mathrm{M}$ U0126 treatment 1 hour prior to CDDP (Fig. 6C). Inhibition of ERK1/2 activation did not detectably change CDDP's induction of p53 in HCT116 3D cultures (Fig. 6C). These findings suggest that ERK1/2 do not play a role in the 3D architecture-mediated p53-dependent decrease in chemosensitivity. \begin{tabular}{llllll}
\multicolumn{4}{c}{ HCT116 3D } & \\
\cline { 1 - 4 } & 1 & 2.5 & 5 & 10 & 20
\end{tabular} L-OHP 24 hour $(\mu \mathrm{g} / \mathrm{ml})$

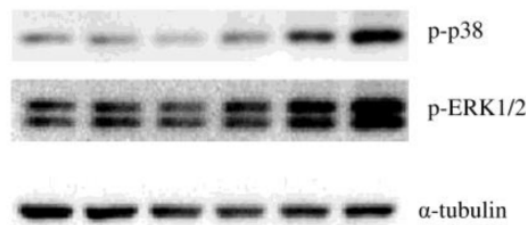

D

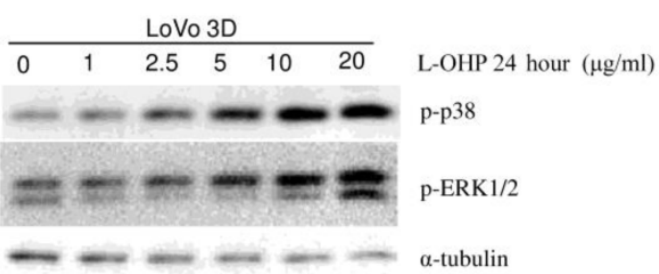

Figure 5. Platinum activates p38 and ERK1/2 (western blot). CDDP activated MAPKs (p38, ERK) in a time-dependent manner in HCT116 3D cultures (A) and LoVo 3D cultures (C). L-OHP activated MAPKs (p38, ERK) in a dose-dependent manner in HCT116 3D cultures (B) and LoVo 3D cultures (D).

A

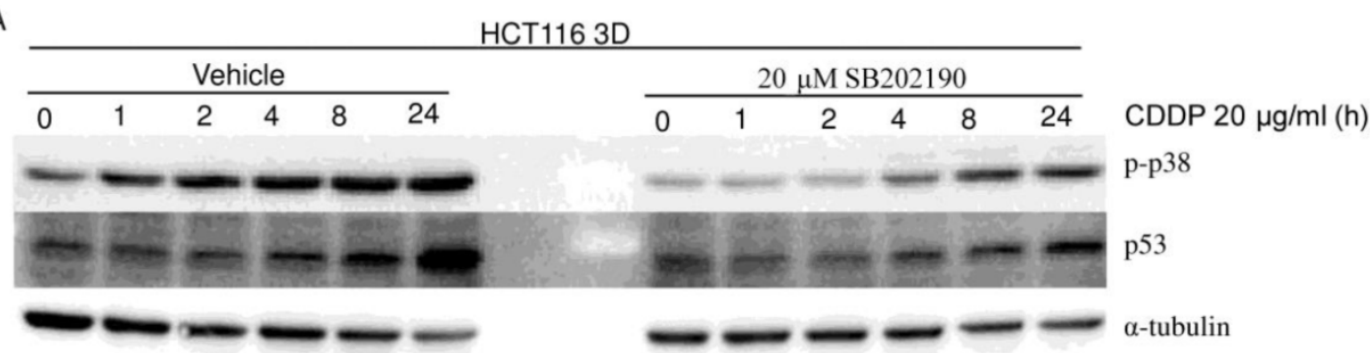

B

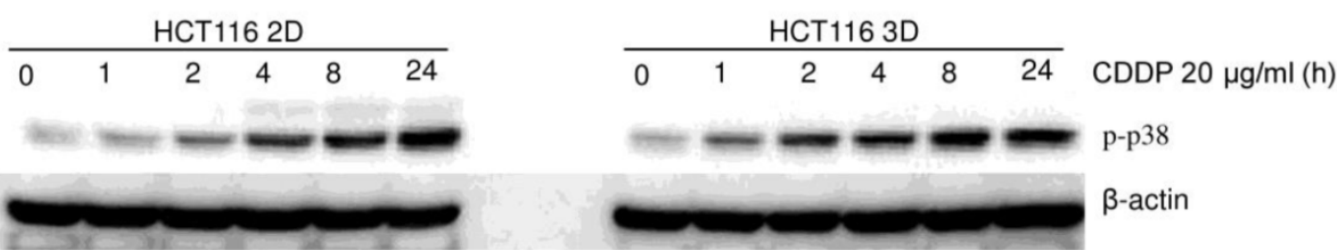

C HCT116 3D
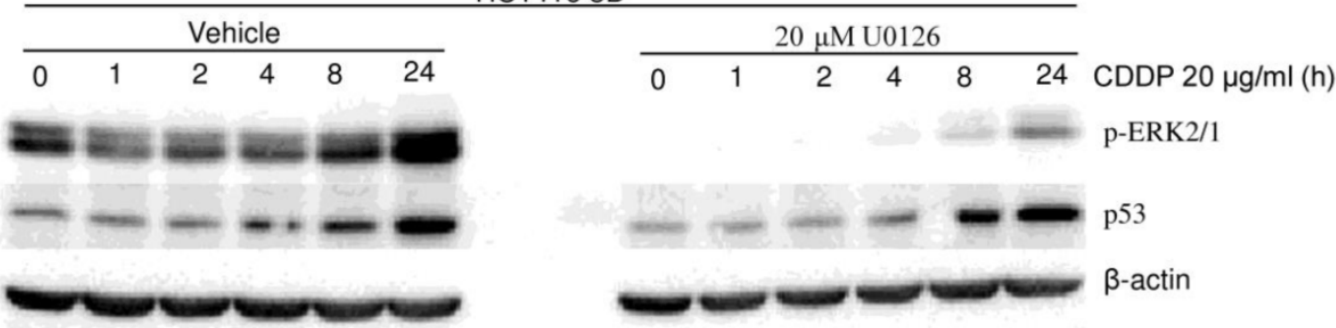

Figure 6. MAPKs (p38 and ERK1/2) do not contribute to the 3D architecture-mediated p53-dependent decrease in chemosensitivity. (western blot). (A) Cells were treated with vehicle or SB202190 1 hour prior to CDDP treatment. CDDP's activation of p38 was remarkably inhibited by SB202190. Inhibition of p38 activation decreased CDDP's induction of p53. (B) There was no detectable difference in P-p38 between $2 D$ cultures and 3D cultures. (C) Cells were treated with vehicle or U0126 1 hour prior to CDDP treatment. CDDP's activation of ERK was remarkably inhibited by U0126. Inhibition of ERK activation did not detectably change CDDP's induction of $\mathrm{p} 53$. 
In summary, although knockdown of p53 decreasing $\mathrm{CDDP}^{\prime} \mathrm{s}$ induction of $\mathrm{p}-\mathrm{JNK} 1 / 2$ and inhibition of p38 activation decreasing $\mathrm{CDDP}^{\prime} \mathrm{s}$ induction of p53 were observed in HCT116 3D cultures, MAPKs (JNK1/2, p38 and ERK1/2) do not play a dominant role in the 3D architecture-mediated p53-dependent decrease in chemosensitivity.

\section{Discussion}

Solid tumor cells in vivo proliferate, survive and response to stimuli in a specific tissue architecture [9-11]. Most in vitro experiments on cancer are performed with 2D cultures [5-6], but 2D cultures do not well recapitulate tissue architecture [9-12]. In 3D cultures, cells adhered to each other within layers of cells, and cell-cell junctions were commonly found (Fig. 1A, B and Fig. S1). Their structures were more similar to those of tumors at avascular stage, avascular tumor regions or micrometastases than $2 \mathrm{D}$ cultures were [8-12]. Therefore, 3D cultures represent a better approximation of solid tumor tissue architecture in vivo [7-12].

Accumulating evidences demonstrate that the architecture of 3D cultures influences cell proliferation (3D cultures contain a core of cells which undergoes senescence), cell survival, nutrient supply, oxygen tension, $\mathrm{pH}$ and so on [7-10, 31-32]. These may play roles in chemosensitivity, more or less [7-10, 31-32]. 3D cultures displayed significantly decreased sensitivity to platinum compared with $2 \mathrm{D}$ cultures (Fig. 1C and D). Tumors in vivo were also reported to present the same characteristics [7-10, 31]. These data suggest that $3 \mathrm{D}$ cultures have the potential to provide predictable results regarding cancer chemotherapeutics [7, 9-12].

Platinum damages DNA by binding to and causing crosslink of DNA to kill cells [19-20]. Following DNA damage, cells respond by activating a DNA-damage response that either leads the cell to repair the lesion, promoting resistance to chemotherapy, or causes cell death by activation of the apoptotic response [19-20, 25]. Protein p53 plays a key role in the response to platinum-induced DNA damage [19-20]. In the current study, platinum treatment increased p53 protein level in a time-dependent and dose-dependent manner. 3D architecture clearly decreased p53 induced by CDDP (Fig. 2). Knockdown of wild-type p53 (Both HCT116 and LoVo contain a stabilized wild-type p53 protein [22-23].) decreased sensitivity to platinum (Fig. 3). These findings indicate that 3D architecture confers decreased chemosensitivity to platinum, by or partly by weaker p53 induction. This may contribute to explain the phenomenon that tumors in vivo display significantly a decreased chemosensitivity than $2 \mathrm{D}$ cultures do [7, 9-10, 31]. At the same time, loss of p53 or mutated p53 is frequently observed in human carcinomas [19, 33-34]. P53 mutations can also inactivate the protein's normal function $[19,34]$. Our results may also contribute to explain that loss of p53 or mutated p53 often acquires oncogenic activities conferring drug resistance and that loss of p53 or mutated $p 53$ is a negative predictor for survival of cancer patients, including colorectal cancer patients [33-36].

The role of apoptosis in the function of p53 in the DNA damage response is well established [19-20, 25]. p53 mediates the DNA-damage response in that p53 can initiate DNA repair; cell-cycle arrest; senescence; and, notably, apoptosis [19-20, 25]. If DNA damage is too great or repair is ineffective, cellular death pathways, such as apoptosis, are stimulated [19-20]. The fact that knockdown of wild-type p53 decreased apoptosis induced by platinum in the present study (Fig. 3) suggests that apoptosis may be involved in the 3D architecture-mediated p53-dependent decrease in sensitivity to platinum.

The MAPK cascade is a critical pathway for human cancer cell apoptosis and resistance to chemotherapy $[5,16,18,27-29]$. There are 4 independent MAPK pathways composed of 4 signaling families: JNK, p38 signaling families, the MAPK/ERK family and the Big MAP kinase-1[28]. In the current study, it was clearly observed that platinum activated JNK1/2, p38 and ERK1/2 in a dose-dependent and time-dependent manner in colon cancer cell 3D cultures (Fig. 4-6). These results are consistent with other reports using 2D cultures or in vivo models [5, 27, 29]. In HCT116 3D cultures, knockdown of p53 decreased CDDP's induction of p-JNK1/2, and inhibition of p38 activation decreased $\mathrm{CDDP}^{\prime} \mathrm{s}$ induction of $\mathrm{p} 53$. However, inhibition of JNK1/2 activation enhanced HCT116 3D cultures' chemosensitivity, and no difference was observed in CDDP's activation of p38 between HCT116 2D cultures and 3D cultures (Fig. 6B). These findings indicate that MAPKs (JNK1/2, p38 and ERK1/2) do not play a dominant role in the $3 \mathrm{D}$ architecture-mediated p53-dependent decrease in chemosensitivity. Further experiments are needed to clarify the complicated mechanism.

\section{Conclusions}

Our results suggest that colorectal cancer cell 3D cultures represent a better approximation of solid tumor tissue architecture, especially cell-cell adhesion, in vivo than 2D cultures do and confer decreased sensitivity to platinum. Weaker p53 induction contributes to the control of colon cancer cells' sensitivity to platinum by 3D architecture. 
Although p53 regulating JNK1/2 and p38 regulating p53 under the condition of CDDP treatment were observed in HCT116 3D cultures, MAPKs (JNK1/2, p38 and ERK1/2) do not play a dominant role in the mechanism of the 3D architecture-mediated p53-dependent decrease in chemosensitivity.

\section{Supplementary Material}

Figure S1. http://www.jcancer.org/v07p0900s1.pdf

\section{Abbreviations}

CDDP: cisplatin; L-OHP: oxaliplatin; 2D: two-dimensional; 3D: three-dimensional; H\&E: Hematoxylin and eosin; sh: small hairpin; shcontrol: control shRNA; WST: Water-soluble tetrazolium salt; TUNEL: the terminal deoxynucleotidyl transferase-mediated nick end-labeling; GAPDH: glyceraldehyde 3-phosphate dehydrogenase; MAPK: mitogen-activated protein kinase; JNK: c-Jun $\mathrm{N}$-terminal kinase; ERK: extracellular signal-regulated kinase.

\section{Acknowledgements}

This work was supported by the National Natural Science Foundation of China (grant no. 81000990), the Natural Science Foundation Project of CQ CSTC (grant no. 2009BB5339) and the Science Foundation of Third Military Medical University for the Young Scholar (grant no. 2009XQN32).

\section{Conflict of interest}

The authors disclose no potential conflicts of interest.

\section{References}

1. Torre LA, Bray F, Siegel RL, Ferlay J, Lortet-Tieulent J, Jemal A. Global cancer statistics, 2012. CA Cancer J Clin. 2015; 65: 87-108.

2. He J, Shin H, Wei X, Kadegowda AK, Chen R, Xie SK. NPC1L1 knockout protects against colitis-associated tumorigenesis in mice. BMC Cancer. 2015; 15: 189

3. Schwartzberg LS, Rivera F, Karthaus M, Fasola G, Canon JL, Hecht JR, et al. PEAK: a randomized, multicenter phase II study of panitumumab plus modified fluorouracil, leucovorin, and oxaliplatin (mFOLFOX6) or bevacizumab plus mFOLFOX6 in patients with previously untreated, unresectable, wild-type KRAS exon 2 metastatic colorectal cancer. J Clin Oncol. 2014; 32: 2240-7.

4. Siegel RL, Miller KD, Jemal A. Cancer statistics, 2015. CA Cancer J Clin. 2015; 65: 5-29.

5. Park GB, Choi Y, Kim YS, Lee HK, Kim D, Hur DY. Silencing of PKCeta induces cycle arrest of $\mathrm{EBV}(+) \mathrm{B}$ lymphoma cells by upregulating expression of p38-MAPK/TAp73/GADD45alpha and increases susceptibility to chemotherapeutic agents. Cancer Lett. 2014; 350: 5-14

6. Godar S, Ince TA, Bell GW, Feldser D, Donaher JL, Bergh J, et al. Growth-inhibitory and tumor- suppressive functions of p53 depend on its repression of CD44 expression. Cell. 2008; 134: 62-73.

7. He JM, Wang FC, Qi HB, Li Y, Liang HJ. Down-regulation of alphav integrin by retroviral delivery of small interfering RNA reduces multicellular resistance of HT29. Cancer Lett. 2009; 284: 182-8.

8. Leithner K, Hrzenjak A, Trotzmuller M, Moustafa T, Kofeler HC, Wohlkoenig $\mathrm{C}$, et al. PCK2 activation mediates an adaptive response to glucose depletion in lung cancer. Oncogene. 2015; 34: 1044-50.

9. Durand RE. Chemosensitivity testing in V79 spheroids: drug delivery and cellular microenvironment. J Natl Cancer Inst. 1986; 77: 247-52.

10. Durand RE. Distribution and activity of antineoplastic drugs in a tumor model. J Natl Cancer Inst. 1989; 81: 146-52.
11. Liang $\mathrm{X}, \mathrm{Xu} \mathrm{X}$, Wang F, Chen $\mathrm{X}, \mathrm{Li} \mathrm{N}$, Wang C, et al. E-cadherin knockdown increases beta-catenin reducing colorectal cancer chemosensitivity only in three-dimensional cultures. Int J Oncol. 2015; 47: 1517-27.

12. Liang $\mathrm{X}, \mathrm{Xu} \mathrm{X}, \mathrm{Wang} \mathrm{F}, \mathrm{Li} \mathrm{N}, \mathrm{He} \mathrm{J}$. E-cadherin increasing multidrug resistance protein 1 via hypoxia-inducible factor-1alpha contributes to multicellular resistance in colorectal cancer. Tumour Biol. 2015.

13. Gomes LR, Vessoni AT, Menck CF. Three-dimensional microenvironment confers enhanced sensitivity to doxorubicin by reducing p53-dependent induction of autophagy. Oncogene. 2015; 34: 5329-40.

14. Casagrande N, Celegato M, Borghese C, Mongiat M, Colombatti A, Aldinucci D. Preclinical activity of the liposomal cisplatin lipoplatin in ovarian cancer. Clin Cancer Res. 2014; 20: 5496-506.

15. Li Y, He J, Zhong D, Li J, Liang H. High-mobility group box 1 protein activating nuclear factor-kappaB to upregulate vascular endothelial growth factor $C$ is involved in lymphangiogenesis and lymphatic node metastasis in colon cancer. J Int Med Res. 2015; 43: 494-505.

16. Bennett BL, Sasaki DT, Murray BW, O'Leary EC, Sakata ST, Xu W, et al. SP600125, an anthrapyrazolone inhibitor of Jun N-terminal kinase. Proc Natl Acad Sci U S A. 2001; 98: 13681-6.

17. Fabian MA, Biggs $W H, 3 r d$, Treiber DK, Atteridge CE, Azimioara MD, Benedetti MG, et al. A small molecule-kinase interaction map for clinical kinase inhibitors. Nat Biotechnol. 2005; 23: 329-36.

18. Zheng B, Fiumara P, Li YV, Georgakis G, Snell V, Younes M, et al. MEK/ERK pathway is aberrantly active in Hodgkin disease: a signaling pathway shared by CD30, CD40, and RANK that regulates cell proliferation and survival. Blood. 2003; 102: 1019-27.

19. Cavallo F, Feldman DR, Barchi M. Revisiting DNA damage repair, p53-mediated apoptosis and cisplatin sensitivity in germ cell tumors. Int J Dev Biol. 2013; 57: 273-80.

20. Zhu Y, Regunath K, Jacq X, Prives C. Cisplatin causes cell death via TAB1 regulation of p53/MDM2/MDMX circuitry. Genes Dev. 2013; 27: 1739-51.

21. Wu ZZ, Sun NK, Chien KY, Chao CC. Silencing of the SNARE protein NAPA sensitizes cancer cells to cisplatin by inducing ERK1/2 signaling, synoviolin ubiquitination and p53 accumulation. Biochem Pharmacol. 2011; 82: 1630-40.

22. Furuwatari C, Yagi A, Yamagami O, Ishikawa M, Hidaka E, Ueno I, et al. A comprehensive system to explore p53 mutations. Am J Clin Pathol. 1998; 110: 368-73.

23. Landers JE, Cassel SL, George DL. Translational enhancement of mdm2 oncogene expression in human tumor cells containing a stabilized wild-type p53 protein. Cancer Res. 1997; 57: 3562-8.

24. Ivashkevich A, Redon CE, Nakamura AJ, Martin RF, Martin OA. Use of the gamma-H2AX assay to monitor DNA damage and repair in translational cancer research. Cancer Lett. 2012; 327: 123-33.

25. Pappano WN, Zhang Q, Tucker LA, Tse C, Wang J. Genetic inhibition of the atypical kinase Wee1 selectively drives apoptosis of p53 inactive tumor cells. BMC Cancer. 2014; 14: 430.

26. $\mathrm{Yu}$ Z, Zhang C, Wang H, Xing J, Gong H, Yu E, et al. Multidrug resistance-associated protein 3 confers resistance to chemoradiotherapy for rectal cancer by regulating reactive oxygen species and caspase-3-dependent apoptotic pathway. Cancer Lett. 2014; 353: 182-93.

27. Sui X, Kong N, Ye L, Han W, Zhou J, Zhang Q, et al. p38 and JNK MAPK pathways control the balance of apoptosis and autophagy in response to chemotherapeutic agents. Cancer Lett. 2014; 344: 174-9.

28. Burotto M, Chiou VL, Lee JM, Kohn EC. The MAPK pathway across different malignancies: a new perspective. Cancer. 2014; 120: 3446-56

29. Koti M, Gooding RJ, Nuin P, Haslehurst A, Crane C, Weberpals J, et al. Identification of the IGF1/PI3K/NF kappaB/ERK gene signalling networks associated with chemotherapy resistance and treatment response in high-grade serous epithelial ovarian cancer. BMC Cancer. 2013; 13: 549.

30. El Fajoui Z, Toscano F, Jacquemin G, Abello J, Scoazec JY, Micheau O, et al. Oxaliplatin sensitizes human colon cancer cells to TRAIL through JNK-dependent phosphorylation of Bcl-xL. Gastroenterology. 2011; 141: 663-73.

31. Kolosenko I, Fryknas M, Forsberg S, Johnsson P, Cheon H, Holvey-Bates EG, et al. Cell crowding induces interferon regulatory factor 9, which confers resistance to chemotherapeutic drugs. Int J Cancer. 2015; 136: E51-61.

32. Mellor HR, Ferguson DJ, Callaghan R. A model of quiescent tumour microregions for evaluating multicellular resistance to chemotherapeutic drugs. Br J Cancer. 2005; 93: 302-9.

33. Sarasqueta AF, Forte G, Corver WE, de Miranda NF, Ruano D, van Eijk R, et al. Integral analysis of $\mathrm{p} 53$ and its value as prognostic factor in sporadic colon cancer. BMC Cancer. 2013; 13: 277.

34. Wei S, Wang H, Lu C, Malmut S, Zhang J, Ren S, et al. Activating Transcription Factor 3 Suppresses the Oncogenic Function of Mutant p53. J Biol Chem. 2014; 289: 8947-59.

35. Stefancikova L, Moulis M, Fabian P, Vasova I, Zedek F, Ravcukova B, et al. Prognostic impact of p53 aberrations for R-CHOP-treated patients with diffuse large B-cell lymphoma. Int J Oncol. 2011; 39: 1413-20.

36. Liu BW, Liu Y, Liu JR, Feng ZX, Liu T. Prognostic effect of p53 expression in patients with completely resected colorectal cancer. Tumour Biol. 2014; 35: 9893-6. 